\title{
Parmesankäse hemmt ACE-System und senkt den Blutdruck!
}

- Hypertonietherapie kann auch ein Genuss sein, wie italienische Wissenschaftler auf dem europäischen Hochdruckkongress berichteten: Der tägliche Verzehr von Grana Padano senkte den Blutdruck in einer Studie in beachtlichem Ausmaß.

Käse-Puristen mögen die Verallgemeinerung der Überschrift verzeihen: Parmigiano-Reggiano und Grana Padano sind natürlich zwei verschiedene Käsesorten, die freilich beide aus genau definierten Regionen Oberitaliens stammen und ähnlich verwendet werden: Gerieben oder in dünne Scheiben geschnitten, veredeln sie manche Speisen, besonders Nudel- und Salatgerichte.

\section{Haltbar wie ein Pharmakon}

Im aktuellen Fall geht es um Grana Padano, einen halbfetten Extrahartkäse aus der PoEbene. Erstmals hergestellt wurde er vor etwa 900 Jahren in der Lombardei, als Zisterzienser-Mönche im Kloster Chiaravalle Milanese daran experimentierten, überschüssige Milch haltbar zu machen. Das Resultat war ein Hartkäse mit körniger Struktur, der in verschiedenen Reifungsstadien angeboten wird und sich bis heute zu einer der beliebtesten Käsesorten der Welt entwickelt hat.

\section{Mittelreifer Käse senkt den Druck am besten}

Der Reifungsgrad des Käses ist auch für den blutdrucksenkenden Effekt von Relevanz. Denn mittelreifer, 9-12 Monate alter Grana Padano enthält die höchste Konzentration bestimmter Tripeptide, die im Zuge des Fermentations-Prozesses durch Lactobacillus helveticus anfallen. „Diese Tripeptide haben einen ACE-hemmenden Effekt", so Studienautor Dr. Giuseppe Crippa, Piacenza.

Dass der Käse aufgrund dieser ACE-inhibitorischen Wirkung tatsächlich den Blutdruck signifikant zu senken vermag, wurde nun in einer randomisierten Studie gezeigt: Die Integration von nur $30 \mathrm{~g}$ Grana Padano in den täglichen Speiseplan reduzierte den Blutdruck bei Patienten mit mäßiger Hypertonie im Vergleich zu einer Kontrollgruppe im Verlauf von acht "Behandlungs"-Wochen signifikant um $8 / 7 \mathrm{mmHg}$, unabhängig von der Art der Blutdruckmessung. An der Studie hatten 30 Verum- und 16-Kontrollpatienten teilgenommen.

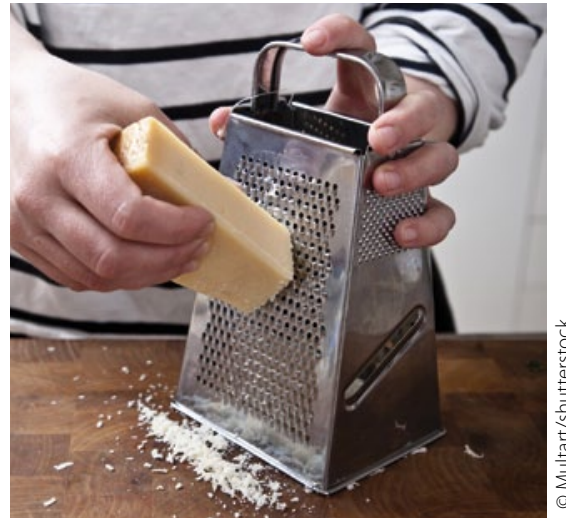

ACE-Inhibitor für Feinschmecker.

\section{Stärkere Blutdrucksenkung als durch} Salzrestriktion

„Patienten unter ACE-Hemmer-/AT ${ }_{1}$-Blocker-Therapie haben wir natürlich ausgeschlossen. Denn zusätzlich zu diesen Medikamenten ist wohl kein blutdrucksenkender Effekt durch den Käse zu erwarten", so Crippa. Nach seinen Ausführungen ist die drucksenkende Wirkung des Käses etwa ebenso groß wie diejenige gängiger Antihypertensiva, und größer als der Effekt einer - kulinarisch bedenklichen - Salzrestriktion. $30 \mathrm{~g}$ Grana Padano enthalten $6 \mathrm{~g}$ Fett und ca. 130-190 mg Salz. Trotz dieser Ingredienzien hielten die Patienten auch ihr Gewicht absolut konstant.

DR. MED. DIRK EINECKE Crippa G. et al. J. Hypertension 2012; 30 (e-Suppl. A):e376

\section{JETZT NOCH SCHNELL MITMACHEN}

\section{Rezepte für gesündere Hypertoniker gesucht}

— Zahlreiche Ärzte haben sich bereits an

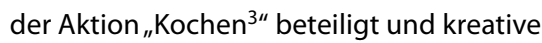
Kochideen eingereicht, auf deren Grundlage ein Kochbuch von Ärzten für Hypertoniker entstehen wird. Bis zum 30. Juni 2012 können auch Sie noch Ihr Rezept einsenden.

"Ich bin begeistert, wie viele leckere und einfache Rezepte mit nur drei Zutaten wir bereits bekommen haben", sagt der Münchner Koch Martin Baudrexel, der die Initiative von Novartis Pharma begleitet. Gesucht werden Rezepte für Gerichte, die aus nur drei Zutaten (plus Gewürze und Basisstoffe wie Butter, Eier, Öl) bestehen, einfach zuzubereiten und originell sind.

„Einfach, stark und individuell“ mit diesem Dreiklang wirbt Novartis nicht nur für einen gesünderen Lebensstil bei Hypertonikern, sondern auch für eine gute Therapietreue in der medikamentösen Hochdruckbehandlung mit dem Präparat Exforge $\mathrm{HCT}^{\circledR}$. Letzteres ermöglicht eine starke Dreifachtherapie mit nur einer Tablette am Tag, welche Valsartan, Amlodipin und HCT enthält und in fünf Dosierungsstärken angeboten wird.
TV-Koch Martin Baudrexel: „Gesundes und kreatives Kochen muss nicht aufwändig sein." 